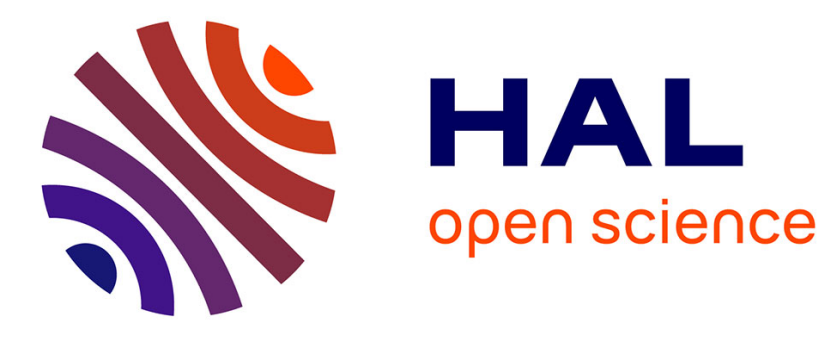

\title{
Perméabilité au gaz de bétons endommagés
}

Vincent Picandet, Abdelhafid Khelidj, Guy Bastian

\section{To cite this version:}

Vincent Picandet, Abdelhafid Khelidj, Guy Bastian. Perméabilité au gaz de bétons endommagés: Interprétation de résultats expérimentaux. Revue Française de Génie Civil , 2002, 6, 10.1080/12795119.2002.9692387 . hal-01007181

\section{HAL Id: hal-01007181 \\ https://hal.science/hal-01007181}

Submitted on 26 Mar 2017

HAL is a multi-disciplinary open access archive for the deposit and dissemination of scientific research documents, whether they are published or not. The documents may come from teaching and research institutions in France or abroad, or from public or private research centers.
L'archive ouverte pluridisciplinaire HAL, est destinée au dépôt et à la diffusion de documents scientifiques de niveau recherche, publiés ou non, émanant des établissements d'enseignement et de recherche français ou étrangers, des laboratoires publics ou privés. 


\title{
Perméabilité au gaz de bétons endommagés Interprétation de résultats expérimentaux
}

\author{
Vincent Picandet - Abdelhafid Khelidj — Guy Bastian \\ Laboratoire de Génie Civil de Nantes Saint-Nazaire, \\ IUT de Saint Nazaire, Département Génie Civil, BP 420 Heinlex, \\ F-44606 Saint Nazaire, cedex \\ vincent.picandet@iutsn.univ-nantes.fr \\ abdelhafid.khelidj@iutsn.univ-nantes.fr \\ guy.bastian@iutsn.univ-nantes.fr
}

RÉSUMÉ. La perméabilité au gaz d'un béton est couramment utilisée pour évaluer ses caractéristiques de durabilité. Cependant, cette mesure est généralement effectuée sur des échantillons sains, n'ayant subi aucun endommagement mécanique. Le but de cette étude expérimentale est d'étudier les effets d'une compression uniaxiale sur la perméabilité au gaz de trois bétons différents : un béton ordinaire et deux bétons hautes performances dont un est renforcé de fibres métalliques. Des charges cycliques ou monotones, comprises entre $60 \%$ et $90 \%$ de la résistance sont appliquées sur des éprouvettes cylindriques 11 x $22 \mathrm{~cm}$. Après déchargement, un disque de béton est découpé dans le milieu de l'éprouvette et est séché en étuve ventilée. Les effets conjugués du séchage et des déformations maximales, subies sous contraintes, sur l'accroissement de la perméabilité sont décrits et analysés.

ABSTRACT. Gas permeability is commonly used to evaluate durability characteristics of concrete. However, these values are often achieved using never stressed nor damaged specimens. The objective of this study is to examine experimentally the effect of axial compressive loading on the permeability of three different types of concrete: Ordinary Concrete, High Performance Concrete, and High Performance Steel Fiber Reinforced Concrete. Monotonic and cyclic loads are applied on 22 x 11-cm diameter specimens. Stress levels vary between $60 \%$ and $90 \%$ of the ultimate strength. After unloading, a disc is extracted from the middle part of the cylinders and is dried in a ventilated oven. The effects of both drying and maximum strain applied during loading are described and analyzed.

MOTS-CLÉS: perméabilité, béton, durabilité, endommagement, microfissure, béton hautes performances, séchage.

KEY WORDS: permeability, concrete, durability, damage, microcracking, high performance concrete, drying. 


\section{Introduction}

La perméabilité d'un béton est, pour de nombreuses applications une caractéristique essentielle du matériau. Pour la mesurer, différentes méthodes ayant recours à un fluide percolant liquide ou gazeux existent. Comme elle est en règle générale faible (inférieure à $10^{-15} \mathrm{~m}^{2}$ ), les mesures directes de perméabilité à l'eau sont délicates à mener, et exigent notamment un temps de mise en œuvre important pour atteindre un régime d'écoulement permanent. Les mesures de perméabilité utilisant un gaz comme fluide de percolation sont par contre, plus rapides et plus souples dans leur mise en œuvre et permettent diverses configurations d'essais. Leur utilisation tend à se développer, tant en laboratoire qu'in-situ [ABE 97], et à petite comme à grande échelle (par exemple, la mise en pression de réservoirs ou d'enceintes de confinement).

Du point de vue de la durabilité, la perméabilité est un facteur majeur, puisqu'elle caractérise l'aptitude du béton à véhiculer des agents agressifs en phase liquide ou gazeuse. Cependant, les mesures de perméabilité considérées sont souvent obtenues sur des bétons sains. Or dans une structure en béton, le matériau est rarement sain. En service, il est soumis à plusieurs types d'agressions extérieures, mécaniques, hydriques et chimiques qui modifient ses caractéristiques initiales. Un couplage existe dans l'évolution des paramètres mécaniques et des paramètres de transferts, tels que la perméabilité. Dans cette étude, le couplage endommagement-perméabilité est évalué en considérant l'effet de l'endommagement mécanique sur la perméabilité du matériau.

Notre objectif a donc été d'évaluer les effets de sollicitations mécaniques extérieures à travers une compression uniaxiale, sur la perméabilité de plusieurs bétons. Dans ce contexte, il est admis que les sollicitations menées au-delà du pic de résistance, en phase post-pic engendrent des dommages importants dans le matériau. Les macrofissures qui apparaissent augmentent alors considérablement la perméabilité [TOR 99]. Dans d'autres configurations de chargements, diverses études ont montré que si des macrofissures traversantes apparaissaient dans le corps d'épreuve, la perméabilité à l'eau croissait notablement [GER 96], [WAN 97]. Dans la phase prépic, c'est à dire pour des chargements inférieurs à $90 \%$ de la résistance, l'endommagement est modéré et se traduit par l'apparition de microfissures diffuses dans le matériau. Dans cette configuration, plusieurs auteurs s'accordent à remarquer un impact mineur sur la perméabilité à l'eau [SAM 92], [HEA 99], tandis qu'une étude antérieure, plus controversée, concluait que dès $40 \%$ de la contrainte ultime, les sollicitations conduisaient à un accroissement très sensible de la perméabilité [KER 91]. Cependant, l'évaluation de l'effet de la microfissuration sur la perméabilité à l'eau est délicate à mener, étant donné les interactions de l'eau avec la matrice cimentaire [HEA 99], surtout pour les bétons hautes performances où l'hydratation n'est pas toujours achevée au moment des essais.

Concernant les mesures de perméabilité au gaz de bétons sollicités en compression uniaxiale, la plupart des études traitent d'essais sous contrainte. Ceux ci ont 
l'avantage de traduire des états plus proches de la réalité mais ont l'inconvénient d'être plus délicats à réaliser. De ce fait, ces études se limitent à des essais sur des mortiers [HEA 97], ou à une configuration imposant un flux de gaz radial dans une éprouvette cylindrique de béton ordinaire [SUG 96]. La présente expérimentation a été menée dans le but d'évaluer les modifications de perméabilité au gaz suivant l'axe de contrainte principal de différents bétons matures, bétons ordinaires et hautes performances. Après déchargement, les éprouvettes ont été rectifiées et ajustées aux dimensions de cellules d'essais d'un perméamètre à charge constante de type Cembureau. Par ailleurs, la teneur en eau des éprouvettes a été soigneusement contrôlée lors du conditionnement. Après avoir examiné les degrés de saturation de chaque éprouvette d'une série d'essais, les perméabilités mesurées sont comparées pour évaluer, en fonction des paramètres mécaniques les plus représentatifs, la variation de la perméabilité. L'influence de la teneur en eau des échantillons lors de tels essais est ensuite analysée afin de déterminer dans quelle mesure la perméabilité au gaz est révélatrice de l'état d'endommagement des bétons.

\section{Programme expérimental}

\subsection{Composition et cure des bétons étudiés}

Trois bétons ont été confectionnés : un béton ordinaire (BO), avec un rapport Eau/Ciment de 0.5 et deux bétons hautes performances (BHP) de compositions similaires avec un rapport $\mathrm{E} / \mathrm{C}$ de 0.3 , mais dont un est renforcé de fibres métalliques (BHPF). Ce dernier contient une fraction volumique de $1 \%$ de fibres métalliques de $30 \mathrm{~mm}$ de longueur et de $0.38 \mathrm{~mm}$ de diamètre. Le détail de chacune des compositions est reporté dans le Tableau 1.

\begin{tabular}{|l|c|c|c|}
\hline Constituants $\left(\mathbf{k g} / \mathbf{m}^{\mathbf{3}}\right)$ & BO & BHP & BHPF \\
\hline Graviers, $12.5-20 \mathrm{~mm}$ & 777 & - & - \\
\hline Gravillons, $4-12.5 \mathrm{~mm}$ & 415 & 1011 & 966 \\
\hline Sable (Boulonnais), $0-5 \mathrm{~mm}$ & 372 & - & - \\
\hline Sable (Loire), 0-4 mm & 372 & 722 & 690 \\
\hline Ciment CPA-CEM I 52.5 & 353 & 400 & 400 \\
\hline Fumée de silice & - & 40 & 40 \\
\hline Fibres métalliques & - & - & 79 \\
\hline Plastifiant & - & 6 & 10 \\
\hline Filler & - & 72.2 & 69 \\
\hline Eau totale & 172 & 140 & 140 \\
\hline Rapport E/C & 0.49 & 0.29 & 0.29 \\
\hline
\end{tabular}

Tableau 1. Composition des bétons étudiés. Mix proportion of test series 
Une vingtaine d'éprouvettes $11 \times 22 \mathrm{~cm}$ issues d'une même gâchée ont été confectionnées pour chacune des séries. Les bétons ont été coulés dans des moules cylindriques et mis en place par vibration. Avant d'être démoulées, les éprouvettes ont été entreposées 24 heures en chambre humide à $20^{\circ} \mathrm{C}$ et $95 \%$ d'humidité relative (HR). Elles ont ensuite été plongées quatre semaines dans l'eau à $20^{\circ} \mathrm{C}$ avant de passer deux jours à $60^{\circ} \mathrm{C}$ dans une étuve non ventilée. A l'issue de cette cure, cinq éprouvettes de chaque composition ont été testées. Les autres éprouvettes ont été stockées dans une salle climatisée $\left(20 \pm 1^{\circ} \mathrm{C}\right.$ et $\left.50 \pm 5 \% \mathrm{HR}\right)$ durant quatre mois avant d'être sollicitées. Les paramètres mesurés sont regroupés dans le Tableau 2.

\begin{tabular}{|l|c|c|c|}
\cline { 2 - 4 } \multicolumn{1}{c|}{} & BO & BHP & BHPF \\
\hline Module d'élasticité $(\mathrm{GPa})$ & 42 & 45 & 46 \\
\hline Résistance en compression, $\sigma_{\mathrm{c}}(\mathrm{MPa})$ & 65 & 110 & 130 \\
\hline Déformation au pic $(\mu \mathrm{m} / \mathrm{m})$ & 2100 & 2950 & 3425 \\
\hline Densité sèche apparente $\left(\mathrm{g} / \mathrm{cm}^{3}\right)$ & 2.34 & 2.35 & 2.41 \\
\hline Porosité ouverte à l'eau $(\%)$ & 12.1 & 9.6 & 10.4 \\
\hline
\end{tabular}

Tableau 2. Propriétés mécaniques et caractéristiques des matériaux. Mechanical characteristics and material properties

\subsection{Sollicitations mécaniques}

Les compressions uniaxiales ont été appliquées à l'aide d'une presse hydraulique programmable asservie en force. Les cycles de charge comprenaient un palier de contrainte minimum de $3.5 \mathrm{MPa}$ et un palier de contrainte maximum, $\sigma_{\max }$, compris entre $60 \%$ et $90 \%$ de la résistance moyenne respective, $\sigma_{\mathrm{c}}$. Un temps de 2 minutes était marqué sur chaque palier, de manière à permettre d'une part le fluage à court terme, se traduisant par la propagation de microfissures dans le matériau, d'autre part, la relaxation du matériau. Par ailleurs, les vitesses de chargement et de déchargement étaient constantes : $0.45 \mathrm{MPa} / \mathrm{s}$. Six éprouvettes de chaque série ont été sollicitées selon le programme présenté dans le Tableau 3.

\begin{tabular}{|l|c|c|c|c|}
\hline Nombre d'éprouvettes & 1 & 1 & 1 & 3 \\
\hline Rapport $\sigma_{\max } / \sigma_{\mathrm{c}}$ & $60 \%$ & $70 \%$ & $80 \%$ & $90 \%$ \\
\hline Nombre de cycles & 10 & 10 & 10 & 1,3 et 10 \\
\hline
\end{tabular}

Tableau 3. Programme de chargement. Loading program

Les déformations ont été mesurées à mi-hauteur de l'éprouvette au moyen d'une cellule extensométrique comprenant trois capteurs LVDT, de $1 \mathrm{~mm}$ de course et de 
$1 \mu \mathrm{m}$ de précision, disposés à $120^{\circ}$ de manière à tenir compte de toutes déformations asymétriques. Les extrémités des éprouvettes sujettes au phénomène de frettage n'ont pas été prises en compte dans les mesures [BOU 99]. Il est supposé que dans la partie médiane de l'éprouvette, l'endommagement produit est uniforme et que les microfissures sont diffuses.

\subsection{Séchage et préparation des échantillons}

Après avoir été sollicitées, les éprouvettes sont découpées à l'aide d'une lame circulaire diamantée de manière à extraire un disque de $50 \mathrm{~mm}$ d'épaisseur. Les disques sont ensuite rectifiés, et leur épaisseur est mesurée avec une précision de 0.1 $\mathrm{mm}$. La surface latérale est enduite de deux couches de résine époxy imperméable afin d'assurer un flux monodirectionnel du gaz traversant les disques lors des mesures de perméabilité.

La perméabilité au gaz d'un béton dont le réseau poreux est saturé en eau n'est pas mesurable. Un drainage, même partiel, est donc nécessaire avant que la perméabilité puisse être mesurée [YSS 97]. Le mode de séchage et le degré de saturation de l'éprouvette ont une influence directe sur la perméabilité au gaz des bétons [JAC 98], [ABB 99]. Dans cette étude, les disques d'une même série ont tous subi le même conditionnement. Ils ont d'abord été soumis à un séchage modéré en étuve ventilée à $60^{\circ} \mathrm{C}$ durant un mois avant que la première mesure de perméabilité ne soit effectuée. Cette première étape de séchage a été sélectionnée car elle n'engendre quasiment aucun dommage pour les bétons tout en permettant un drainage suffisant pour initier la percolation du gaz à travers les échantillons. Cependant, afin d'assurer une homogénéisation des degrés de saturation entre les éprouvettes d'une même série, la procédure de séchage se poursuit durant six mois supplémentaires, en plusieurs étapes, à des températures plus élevées (voir Tableau 4).

\begin{tabular}{|c|c|c|}
\hline & BO & BHP \& BHPF \\
\hline Etape de séchage $\mathrm{n}^{\circ} 1$ & \multicolumn{2}{|c|}{1 mois à $60^{\circ} \mathrm{C}$} \\
\hline Etape de séchage $n^{\circ} 2$ & +1 mois à $80^{\circ} \mathrm{C}$ & +1 mois à $105^{\circ} \mathrm{C}$ \\
\hline Etape de séchage $n^{\circ} 3$ & \multicolumn{2}{|c|}{+2 mois $105^{\circ} \mathrm{C}$} \\
\hline Etape de séchage $n^{\circ} 4$ & \multicolumn{2}{|c|}{+3 mois $105^{\circ} \mathrm{C}$} \\
\hline
\end{tabular}

Tableau 4. Procédure de séchage. Drying procedure

Le séchage, appliqué par étapes de températures croissantes, limite les gradients hydriques et thermiques dans l'échantillon. Cependant, le préconditionnement à des températures élevées $\left(105^{\circ} \mathrm{C}\right)$ endommage la microstructure des bétons et accroît artificiellement leur perméabilité au gaz [YSS 97]. Le problème est que, à des températures inférieures, les durées de stabilisation et d'homogénéisation des degrés 
de saturation des échantillons sont très longues. Par ailleurs, il a été constaté qu'une durée de séchage importante, surtout pour les BHP, permet par la suite de réduire les dispersions des mesures de perméabilité apparente [QUE 97].

Notons que, par la suite, la perméabilité est seulement étudiée en fonction de l'endommagement mécanique lié aux sollicitations extérieures. L'endommagement lié aux séchages poussés est considéré comme identique pour chaque échantillon d'une même série. Après chaque étape de séchage, les échantillons sont maintenus durant $48 \mathrm{~h}$ dans un dessiccateur à $20^{\circ} \mathrm{C}$ avant de procéder aux mesures de perméabilité.

\subsection{Mesure de la perméabilité au gaz}

La perméabilité au gaz a été mesurée à l'aide d'un perméamètre à charge constante de type Cembureau [KOL 89]. Un schéma de principe du perméamètre utilisé est donné Figure 1.

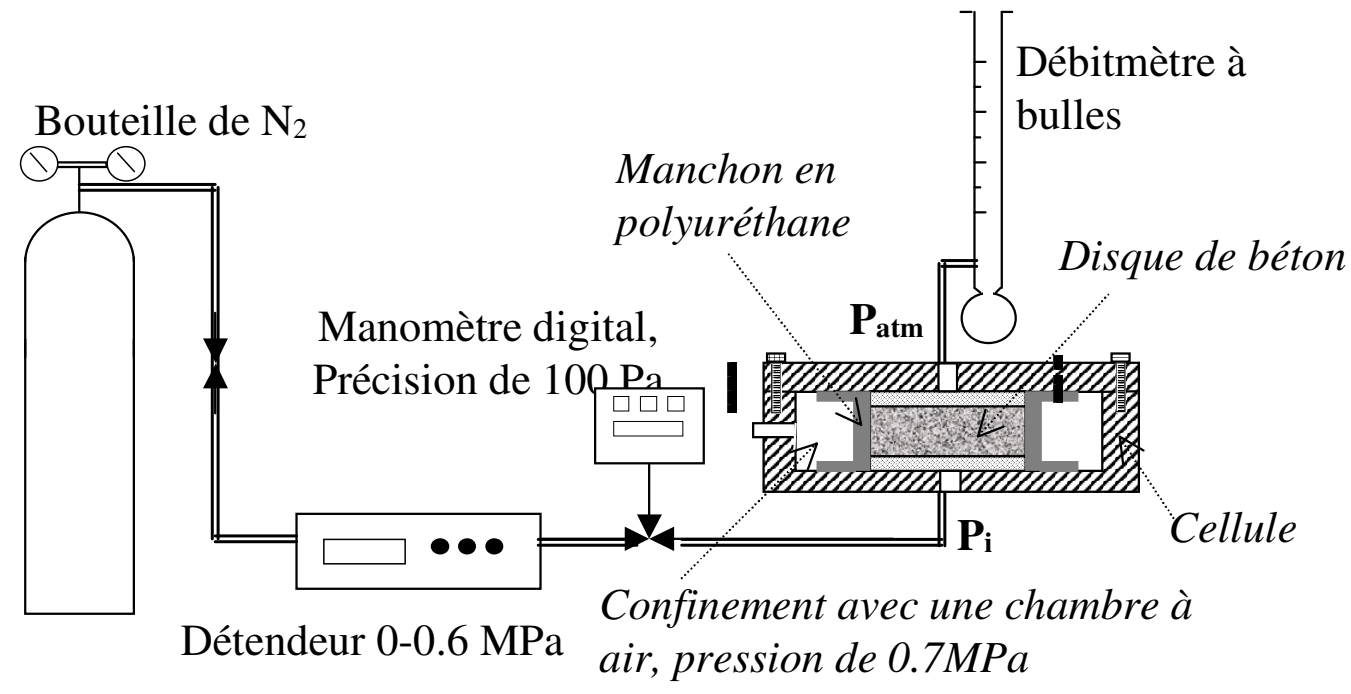

Figure 1. Schéma du dispositif expérimental: perméamètre à charge constante. Schematic layout of the experimental setup : constant head permeameter

La pression relative $\left(\mathrm{P}_{\mathrm{i}}-\mathrm{P}_{\mathrm{atm}}\right)$ appliquée à l'échantillon a été mesurée avec un manomètre digital de $100 \mathrm{~Pa}$ de précision. Les mesures de perméabilité ont eu lieu en salle climatisée à $\left(20 \pm 1^{\circ} \mathrm{C}\right)$ avec de l'azote pour gaz percolant. Chaque test comprenait cinq mesures relatives aux pressions différentielles appliquées suivantes : $0.05,0.1,0.15,0.2,0.3 \mathrm{MPa}$. Un temps suffisamment long est requis entre deux mesures afin que l'écoulement de gaz puisse s'établir en régime permanent à travers l'échantillon. Cette condition était supposée vérifiée lorsque les débits mesurés à 10 mn d'intervalle ne différaient pas de plus de $2 \%$. 
Pour chaque pression différentielle, le coefficient de perméabilité apparent $\mathrm{k}_{\mathrm{A}}\left(\mathrm{m}^{2}\right)$ est calculé à partir de l'équation [1] qui est une adaptation de la relation de HagenPoiseuille, pour un fluide visqueux compressible en écoulement laminaire à travers un milieu poreux.

$$
\mathrm{k}_{\mathrm{A}}=\frac{\mathrm{Q}}{\mathrm{A}} \cdot \frac{2 \mu \cdot \mathrm{L} \cdot \mathrm{P}_{\mathrm{atm}}}{\left(\mathrm{P}_{1}^{2}-\mathrm{P}_{\mathrm{atm}}{ }^{2}\right)}
$$

- L : épaisseur de l'échantillon (m)

- A : section $\left(\mathrm{m}^{2}\right)$

- Q : débit volumique de gaz à pression atmosphérique $\left(\mathrm{m}^{3} / \mathrm{s}\right)$

- $\mu$ : viscosité dynamique $\left(1,76.10^{-5} \mathrm{~Pa}\right.$.s pour le diazote à $\left.20^{\circ} \mathrm{C}\right)$

- $\mathrm{P}_{\mathrm{i}}$ : pression absolue d'injection $(\mathrm{Pa})$

- $\mathrm{P}_{\mathrm{atm}}$ : pression aval, pression atmosphérique $(\mathrm{Pa})$

En fait, la percolation d'un gaz dans un milieu poreux tel que le béton peut être considérée comme résultant de deux modes d'écoulement distincts : un écoulement visqueux et un écoulement par glissement ou effet de Knudsen. Ce dernier écoulement prend une importance particulière lorsque le réseau poreux est relativement fin, c'est à dire lorsque le diamètre moyen des capillaires traversés par le flux gazeux est de l'ordre de grandeur du libre parcours moyen des molécules de gaz. Différentes méthodes existent pour calculer cet écoulement par glissement, mais la méthode la plus répandue est celle de Klinkenberg, traduite par l'équation [2]. Elle permet de déterminer la perméabilité intrinsèque $\mathrm{k}_{\mathrm{V}}$, relative uniquement aux écoulements visqueux, à partir de plusieurs mesures de perméabilité apparente à différentes pressions.

$$
\mathrm{k}_{\mathrm{A}}=\mathrm{k}_{\mathrm{V}}\left(1+\frac{\beta}{\mathrm{Pm}_{\mathrm{m}}}\right)
$$

- $\mathrm{P}_{\mathrm{m}}$ :pression moyenne, $\mathrm{P}_{\mathrm{m}}=\left(\mathrm{P}_{\mathrm{i}}+\mathrm{P}_{\mathrm{atm}}\right) / 2$

- $\beta$ : coefficient de Klinkenberg $(\mathrm{Pa})$

$\mathrm{k}_{\mathrm{V}}$ est la valeur limite de la perméabilité apparente $\mathrm{k}_{\mathrm{A}}$ lorsque la pression moyenne du fluide tend vers l'infini, c'est à dire lorsque le gaz tend vers une phase condensée (liquide). La méthode consiste donc à effectuer une régression linéaire des différentes mesures de perméabilité apparente selon l'inverse de la pression moyenne. Elle est illustrée en Figure 2 à partir de mesures réalisées sur un disque de BO. Pour tous les essais, les mesures relatives aux cinq pressions différentielles distinctes se corrèlent bien, avec un coefficient supérieur 0.99 .

Il est à noter que la perméabilité intrinsèque, $\mathrm{k}_{\mathrm{V}}$, doit son qualificatif uniquement au fait d'être significative du seul écoulement visqueux. Sa valeur dépend encore du degré de saturation du matériau lors des essais. $\mathrm{k}_{\mathrm{V}}$ augmente lorsque la teneur en eau diminue [ABB 99]. 
D'après l'équation [2], le débit des écoulements par glissement $\mathrm{Q}_{\text {gliss }}$, est proportionnel au terme $\mathrm{k}_{\mathrm{v}} \beta / \mathrm{P}_{\mathrm{m}}$, tandis que le débit des écoulements visqueux $\mathrm{Q}_{\mathrm{visq}}$ est proportionnel à $\mathrm{k}_{\mathrm{V}}$ (Figure 2). Pour une pression différentielle définie, l'équation [3] donne le rapport de ces débits.

$$
\frac{Q_{\text {gliss }}}{Q_{\text {visq }}}=\frac{\beta}{P_{m}}
$$

Si la pression moyenne augmente, le libre parcours moyen des molécules de gaz diminue et la part des écoulements par glissement décroît. Lorsque la pression moyenne $\mathrm{P}_{\mathrm{m}}$ est égale à $\beta$, le débit dû aux écoulements visqueux équivaut au débit dû aux écoulements par glissement. Par ailleurs, si le rayon moyen des pores traversés par le flux de gaz augmente, la part des écoulements par glissement décroît et le rapport donné par l'équation [3] proportionnel à $\beta$ diminue. La valeur de $\beta$ est donc significative de la finesse du réseau poreux emprunté par le gaz lors de son écoulement. Cette valeur est intrinsèque au matériau, pour un état de saturation donné.

Dans le cadre de la plupart des mesures de perméabilité, une seule pression différentielle est appliquée, ou une seule pression moyenne est considérée (cas des essais à charge variable par exemple). Dans ces cas de mesures, seule la valeur $\mathrm{k}_{\mathrm{A}}$

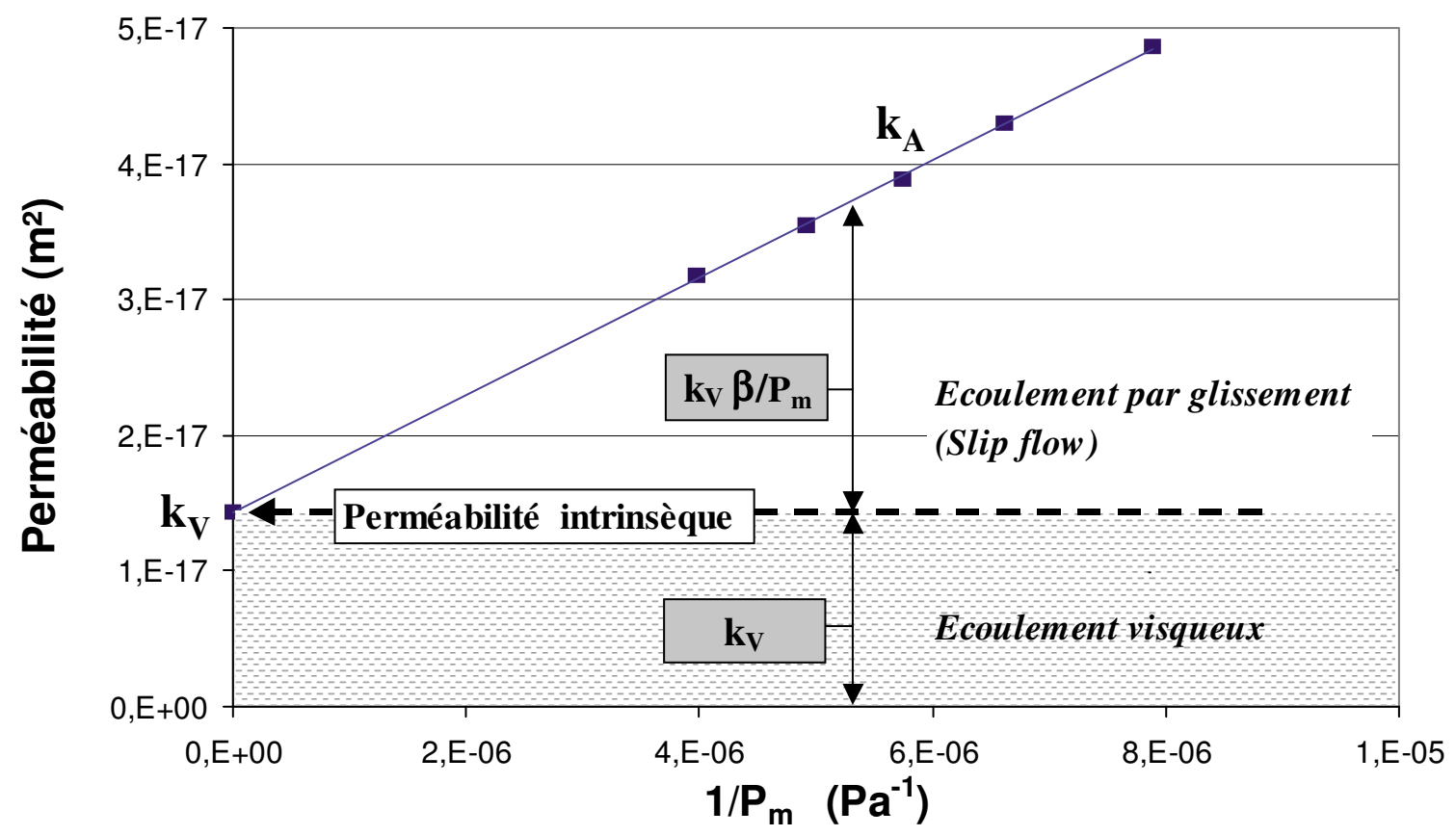

Figure 2. Exemple et interprétation d'un graphe $k_{A}=F\left(1 / P_{m}\right)$ obtenu à partir de mesures expérimentales pour un $B O$. Example and interpretation of a graph $\mathrm{k}_{\mathrm{A}}=F\left(1 / \mathrm{P}_{\mathrm{m}}\right)$ obtained from experimental measurements for a BO. 
(équation [1]) est considérée. Or les écoulements par glissement qui ont lieu lors de la percolation sont aussi pris en compte dans les mesures et conduisent alors à une surestimation de la perméabilité. Cette surestimation diminue avec l'augmentation de la pression moyenne, mais demeure non négligeable si la pression d'injection est inférieure à $1 \mathrm{MPa}$.

Par la suite, cette étude expérimentale tient compte des perméabilités relatives aux écoulements visqueux $\mathrm{k}_{\mathrm{V}}$ ainsi que des évaluations du coefficient $\beta$ (équation [2]). Les perméabilités apparentes $\mathrm{k}_{\mathrm{A}}$ sont ponctuellement considérées à titre de comparaison.

\section{Comparaison des perméabilités}

\subsection{Degrés de saturation après chaque étape de séchage}

Le séchage en étuve ventilée à $105^{\circ} \mathrm{C}$ a été mené sur une période totale de six mois en étuve pour les BHP et de cinq mois pour le BO. A l'issue du séchage, la variation de masse des disques à sept jours d'intervalle n'excédait pas $0.07 \%$ pour les BHP et $0.03 \%$ pour le $\mathrm{BO}$. Après la dernière étape de séchage, il a été considéré que les disques avaient atteint leur masse sèche de référence. Par ailleurs, des échantillons, stockés dans l'eau à $20^{\circ} \mathrm{C}$ en permanence depuis le second jour suivant leur coulage, sont utilisés pour déterminer les masses volumiques des bétons à saturation et pour calculer leurs porosités ouvertes à l'eau présentées dans le Tableau 2. A partir de ces valeurs, les degrés de saturation de chaque disque ont été calculés après les trois premières étapes de séchage (voir Figure 3).

La difficulté du séchage préalable à une mesure de perméabilité, est d'obtenir des degrés de saturation qui soient uniformes, d'une part entre chaque disque à comparer, et d'autre part au sein même des échantillons. Or le premier effet de l'endommagement observable sur les paramètres de transfert du matériau apparaît lors du séchage : la diffusivité hydrique est amplifiée et la cinétique de séchage est plus rapide dans les échantillons les plus endommagés. La Figure 3 montre en effet qu'après la première étape de séchage, les degrés de saturation des disques d'une même série sont plus faibles pour les disques extraits des éprouvettes les plus sollicitées. Cet effet est surtout visible après un séchage modéré et est plus marqué pour les BHP que pour le BO dont le séchage est plus rapide.

Après l'étape de séchage $\mathrm{n}^{\circ} 3$ et, a fortiori, après l'étape de séchage $\mathrm{n}^{\circ} 4$, les teneurs en eau de chaque éprouvette d'une série tendent vers une même valeur et les écarts sont minimes (les degrés de saturation sont inférieurs à $2 \%$ ). Il a alors été considéré que les différences engendrées dans la cinétique de séchage des disques n'avaient plus d'influence, notamment sur les mesures de perméabilité suivantes. 


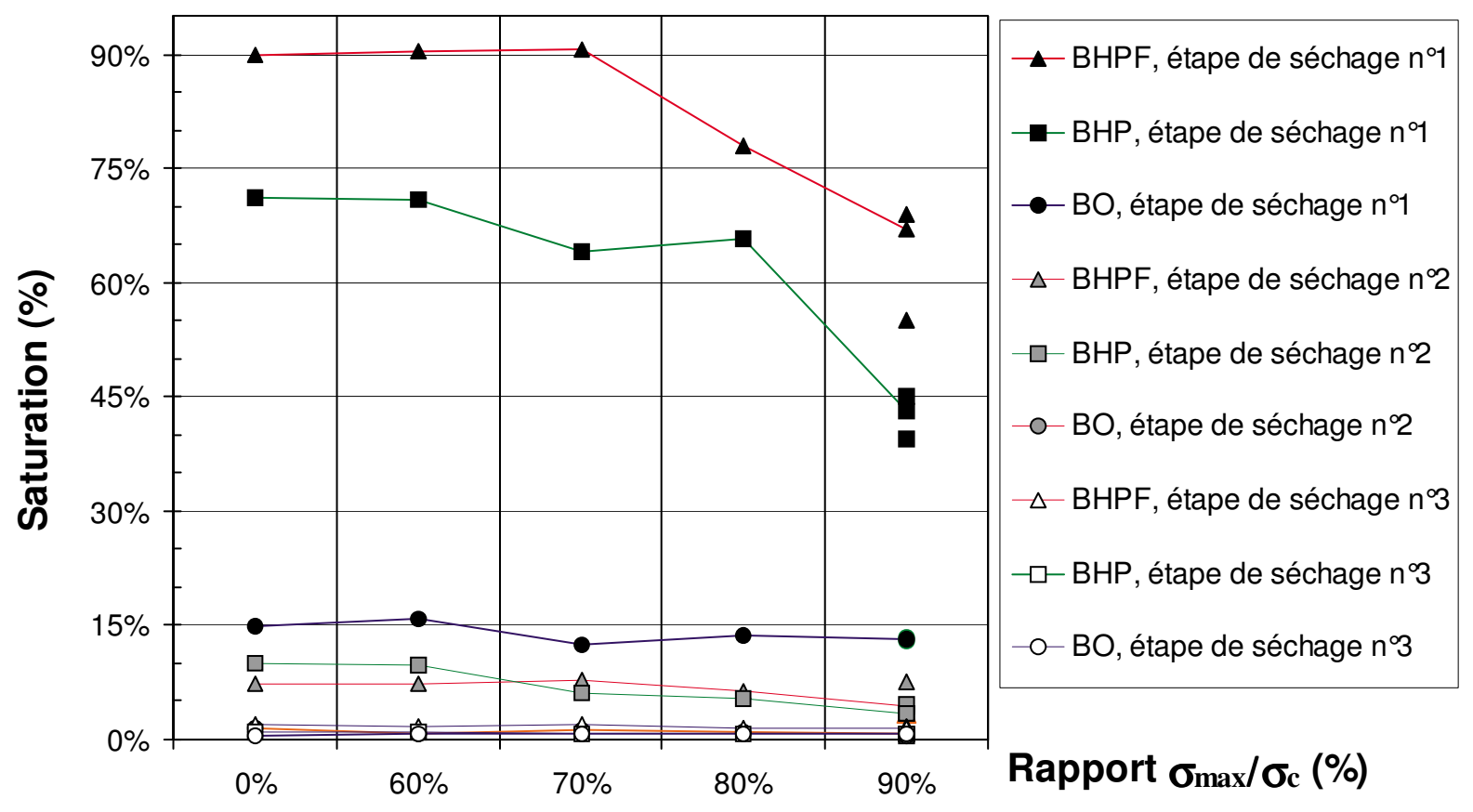

Figure 3. Degré de saturation des disques après les trois premières étapes de séchage. Degree of water saturation of discs after the first three drying stages

\subsection{Partition du flux gazeux dans un échantillon endommagé}

Après chaque étape de séchage, la perméabilité de chaque béton sain a été déterminée à partir de quatre disques. La moyenne de ces quatre valeurs est

Réseau poreux initial (faible perméabilité)

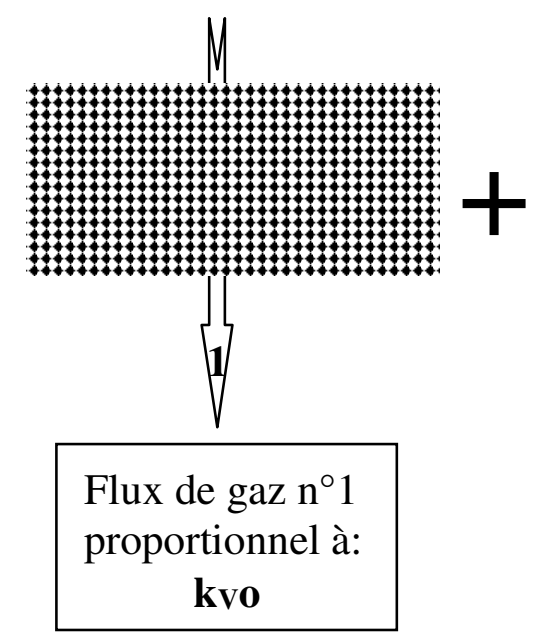

Réseau de fissures et de nouveaux pores connectés, dans un milieu imperméable
Milieu poreux

fissuré

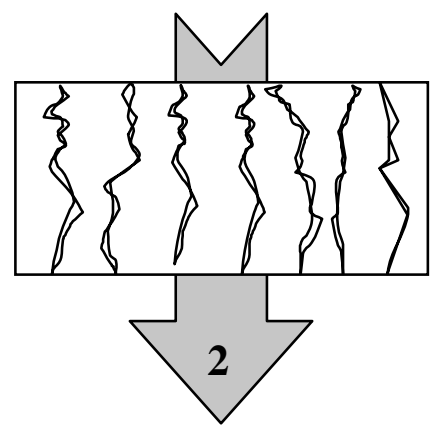

Flux de gaz $n^{\circ} 2$ proportionnel à:

$$
\operatorname{kv}(d) \text { - kvo }
$$

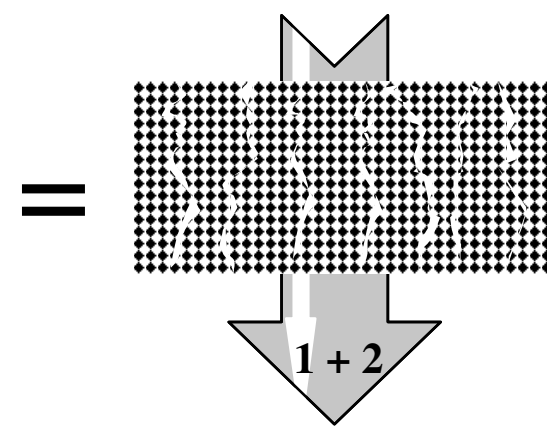

Flux de gaz total proportionnel à: $\operatorname{kv}(\mathbf{d})$

Figure 4. Hypothèse de partition du flux de gaz à travers un échantillon endommagé. Hypothesis of gas flow partition through a damaged sample 
considérée comme la perméabilité initiale $\mathrm{k}_{\mathrm{V}} \mathrm{O}$, qui sert de valeur de référence aux comparaisons ultérieures. Les écarts observés sont de l'ordre de $\pm 20 \%$ et décroissent avec le séchage. Dans les conditions respectives de séchage, la perméabilité des disques extraits des éprouvettes endommagées, est notée $\mathrm{k}_{\mathrm{V}}(\mathrm{d})$.

Le flux gazeux percolant par écoulement visqueux à travers un échantillon endommagé peut alors être divisé en deux :

- Le flux de gaz percolant à travers le réseau poreux non endommagé mécaniquement. C'est le flux de gaz minimum, percolant à travers les disques de référence et proportionnel à la valeur de $\mathrm{k}_{\mathrm{V}} \mathrm{O}$.

- Le flux de gaz empruntant le réseau de microfissures et des pores nouvellement connectés au sein du béton. C'est la différence entre le flux de gaz total percolant à travers un même disque endommagé et le flux de gaz précédent. Ce flux de gaz empruntant uniquement les chemins de percolation relevant de l'endommagement du matériau est proportionnel à l'accroissement de perméabilité absolu : $\mathrm{k}_{\mathrm{V}}(\mathrm{d})-\mathrm{k}_{\mathrm{V}} \mathrm{o}$ (voir Figure 4).

\section{Effets des sollicitations mécaniques sur la perméabilité}

Parmi les différents paramètres enregistrés durant la phase de compression, la déformation maximale sous contrainte est le paramètre qui traduit un lien direct avec les modifications de la perméabilité du béton après déchargement.

\subsection{Relation entre la déformation maximale subie et la perméabilité}

Pour chaque série, la déformation sous contrainte maximale a été atteinte pour l'une des trois éprouvettes testées à $90 \%$ de fc, indépendamment du nombre de cycles. En fait, à ce niveau de contrainte, il existe une différence sensible de comportement d'une éprouvette à une autre, et l'endommagement produit est variable même si les écarts constatés lors des mesures de la résistance de chaque série étaient modérés, (inférieurs à 5\% sur cinq éprouvettes). Les déformations subies durant les sollicitations apparaissent par contre comme révélatrices du comportement physique des éprouvettes vis à vis du chargement. En fonction de ce dernier paramètre, les perméabilités intrinsèques, $\mathrm{k}_{\mathrm{V}}$, des disques extraits des éprouvettes, après les étapes de séchage $\mathrm{n}^{\circ} 1$ et $\mathrm{n}^{\circ} 4$ sont reportées respectivement dans la Figure 5 et la Figure 6.

Après un mois de séchage à $60^{\circ} \mathrm{C}$ en étuve ventilée, le degré de saturation des BHP est toujours supérieur à 70\% (voir Figure 3 ). Cette première étape de séchage a pour but de drainer partiellement les échantillons de BHP en quantité nécessaire pour pouvoir initier la percolation de gaz à travers l'échantillon et procéder à des mesures de perméabilité. Néanmoins, pour les échantillons non endommagés, la teneur en 


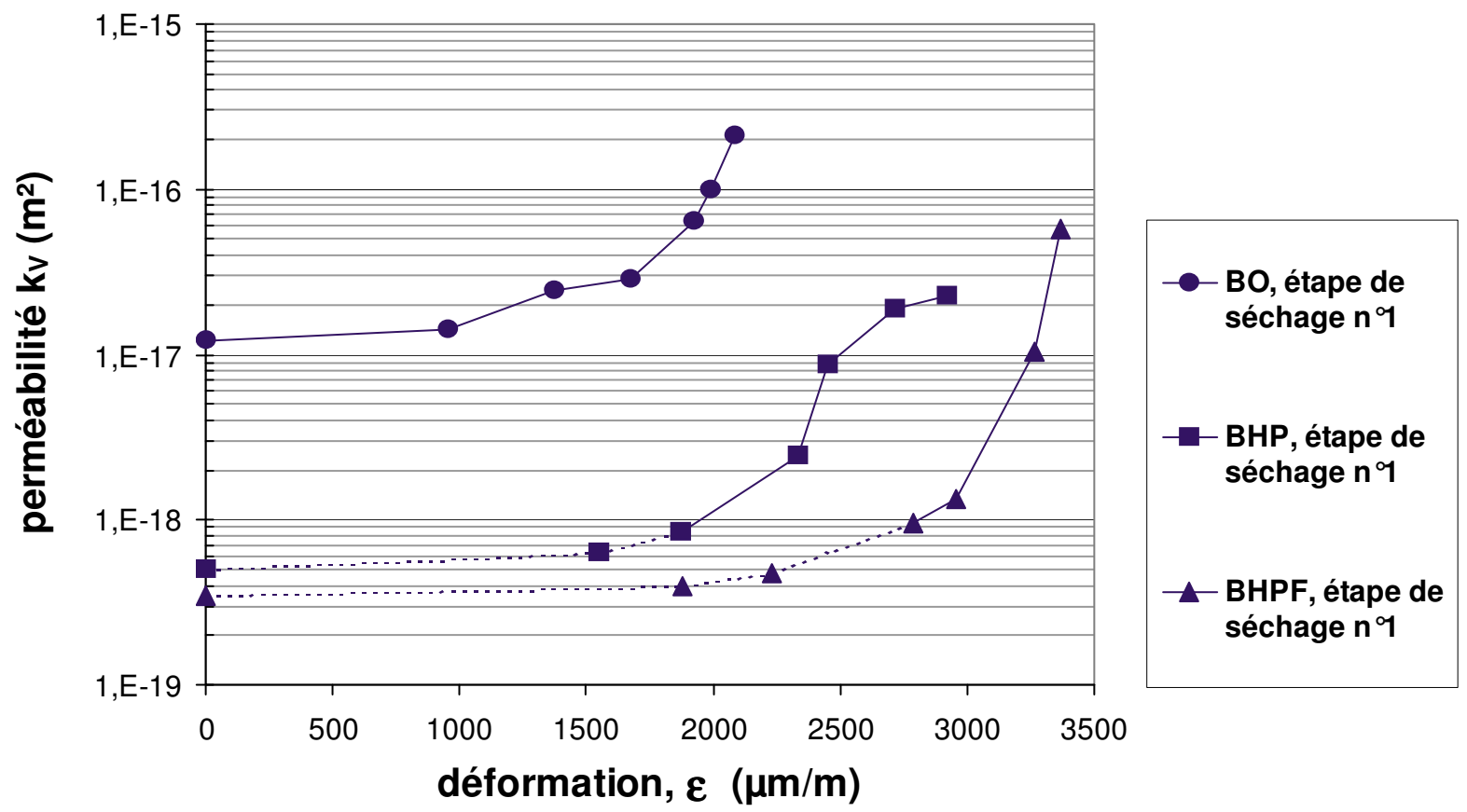

Figure 5. Perméabilité au gaz des disques après la première étape de séchage selon les déformations maximales subies. Gas permeability of discs after the first drying stage versus the maximum applied strain

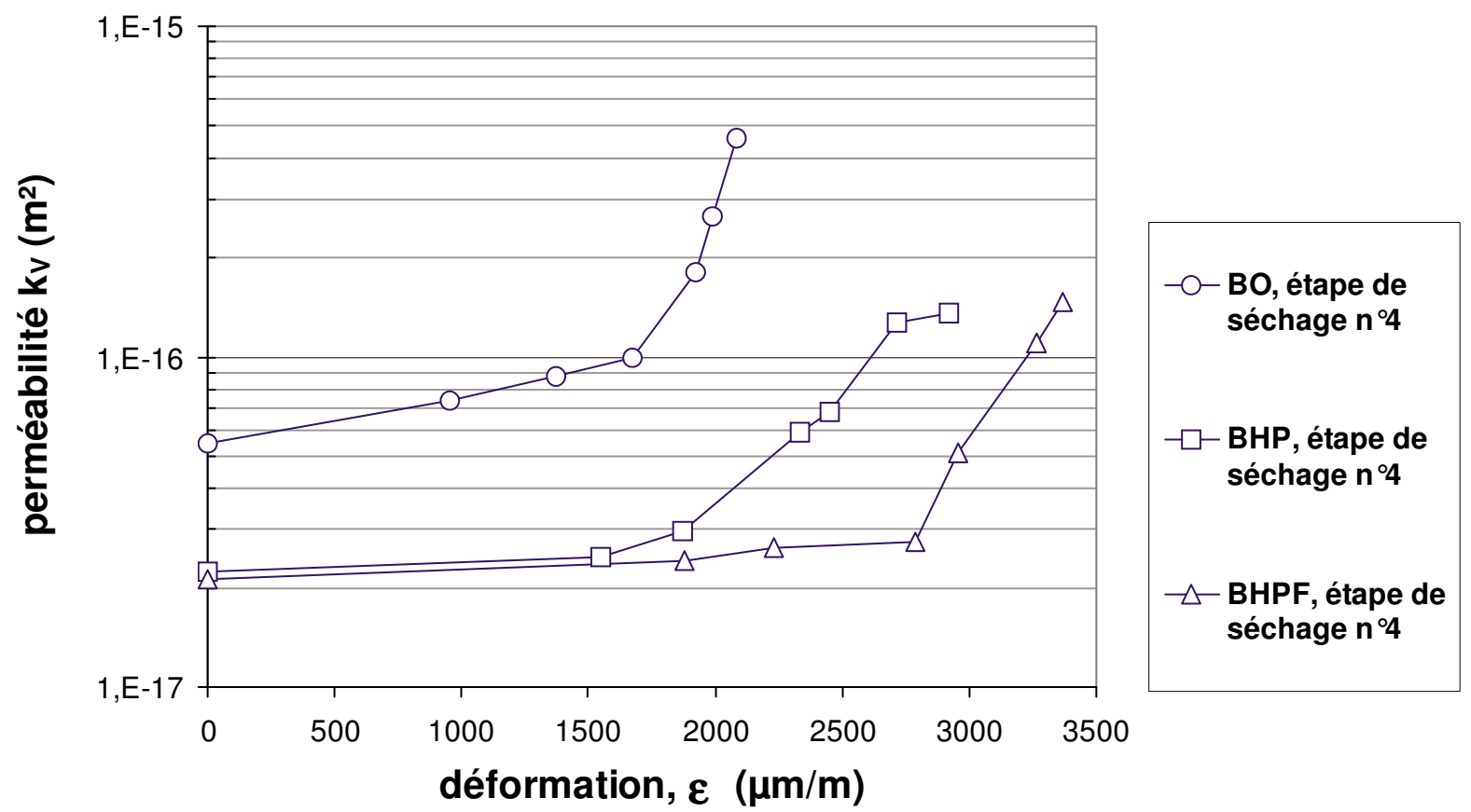

Figure 6. Perméabilité au gaz des éprouvettes après la dernière étape de séchage. Gas permeability of discs after the last drying stage versus the maximum applied strain 
eau demeure toujours importante (cf. §3.1) et la perméabilité faible (inférieure à $5.10^{-19} \mathrm{~m}^{2}$ ). Pour de faibles pressions d'injection, le flux de gaz n'est pas suffisant pour permettre des mesures de perméabilité significatives. La perméabilité intrinsèque a donc été majorée par une mesure de perméabilité apparente effectuée pour une pression d'injection de $3.5 \mathrm{MPa}$. Une ligne pointillée relie ces points de mesures dans la Figure 5, ainsi que dans les figures suivantes.

Concernant le comportement des différents types de bétons, il apparait clairement que si une même déformation a été appliquée, la perméabilité du BO est toujours supérieure à celle des BHP après chaque étape de séchage. Les BHP supportent des déformations plus grandes, mais après un séchage modéré leur rapport d'accroissement de perméabilité est plus grand (voir Figure 5). Le BHP fibré supporte les contraintes les plus élevées et en conséquence les déformations les plus importantes. Le rôle des fibres intervient donc en premier lieu sur les caractéristiques mécaniques, et, dans le cas où des déformations croissantes sont appliquées, intervient en second lieu en différant l'augmentation de perméabilité des BHP après déchargement.

\subsection{Accroissement de la perméabilité en fonction du rapport $\varepsilon / \varepsilon_{c}$}

Afin d'établir une comparaison de comportement entre les bétons, les déformations maximales sous contrainte $\varepsilon$ sont rapportées à la déformation au pic, $\varepsilon_{\mathrm{c}}$, du béton considéré. Cette déformation au pic a été évaluée avec une variation de $\pm 3 \%$, et les valeurs sont données dans le Tableau 2 (§ 2.1).

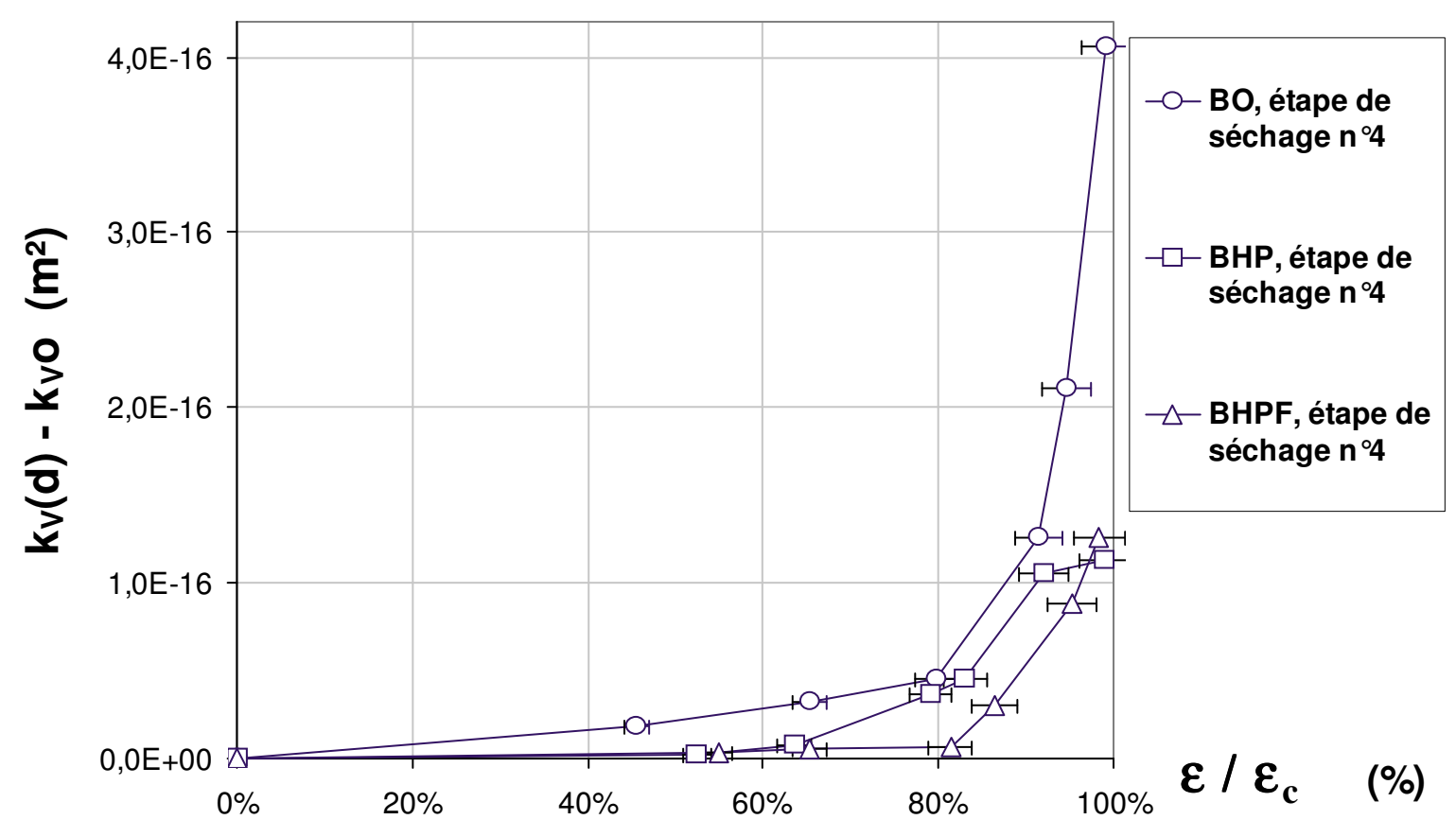

Figure 7. Accroissement absolu de la perméabilité de chaque béton après la dernière étape de séchage en fonction du rapport de déformation $\mathcal{E} \mathcal{E}_{c}$. Absolute 
increase in permeability of each concrete after the last drying stage versus the strain ratio $\varepsilon / \varepsilon_{\mathrm{c}}$

La Figure 7 montre que pour chacun des trois bétons étudiés, il existe un seuil de déformation se situant autour de $80 \%$ de $\varepsilon_{\mathrm{c}}$ à partir duquel la perméabilité augmente sensiblement. Ce seuil doit correspondre à la formation d'un réseau de microfissures connectées réparties dans l'échantillon. Ces fissures, une fois créées, ne se referment pas complètement après déchargement [SAM 92, HEA 99]. Un tel accroissement de la perméabilité a aussi été observé lors d'essais sous contrainte autour d'un état de contrainte-déformation très proche [HEA 97, SUG 96]. Au-delà de ce seuil, l'endommagement créé dans les bétons a une influence sensible sur la perméabilité , et irréversible puisque les fissures demeurent après déchargement.

\subsection{Considérations microstructurales.}

Pour observer la microfissuration produite, les sections des disques les plus endommagés ont été examinées à l'aide d'un vidéo-microscope en lumière rasante avec des grossissements compris entre 100x et 200x. Par cette méthode, des microfissures de $3 \mu \mathrm{m}$ d'ouverture moyenne et de quelques millimètres de longueur peuvent être détectées [PIC 00]. Des disques ont aussi été imprégnés de colorant, mais aucune observation n'a permis de détecter clairement un réseau de fissuration ou la présence de fissures importantes. Seules quelques fissures diffuses autour des granulats ont été détectées. Leur ouverture moyenne était alors de $3 \mu \mathrm{m}$ et leur longueur inférieure à $5 \mathrm{~mm}$. Ces microfissures paraissaient être uniformément distribuées sur la section de l'éprouvette, sans interconnections particulières dans ce plan d'observation.

Par ailleurs, les mesures de perméabilité pour cinq pressions différentielles permettent de déterminer le coefficient $\beta$ (cf. \$2.4) avec une précision estimée à $\pm 10 \%$. Les valeurs de $\beta$ diminuent avec les déformations subies par le matériau durant le chargement (Figure 8). Cette diminution révèle que l'écoulement global du gaz à travers un disque endommagé a lieu principalement par des chemins de percolation de section moyenne plus importante. Cela implique donc que les contraintes exercées ont provoqué des dégradations au niveau de la microstructure du matériau. Les microfissures engendrées, avec l'aide éventuelle des pores qu'elles connectent, créent alors un chemin d'écoulement préférentiel.

Dans le cadre d'essais de perméabilité pour une seule pression différentielle, la mesure de $\mathrm{k}_{\mathrm{A}}$ conduit a une surestimation relative de la perméabilité du matériau plus importante pour un béton sain que pour un béton endommagé. D'après l'équation [3], pour une pression d'injection relative de $0.1 \mathrm{MPa}$ après la dernière étape de séchage, les écoulements par glissement, pris en compte dans la mesure de $\mathrm{k}_{\mathrm{A}}$, représentent plus de $120 \%$ de l'écoulement visqueux pour un BO sain contre moins de $30 \%$ pour un BO endommagé. Donc, entre un béton sain et un béton endommagé, le rapport d'accroissement de la perméabilité apparente $\mathrm{k}_{\mathrm{A}}$ (pour une 
pression différentielle unique) est inférieur au rapport d'accroissement relatif de la perméabilité intrinsèque $\mathrm{k}_{\mathrm{V}}$. Le rapport d'accroissement de perméabilité apparente dû à l'endommagement est surtout diminué lorsque que la pression d'injection considérée est faible.

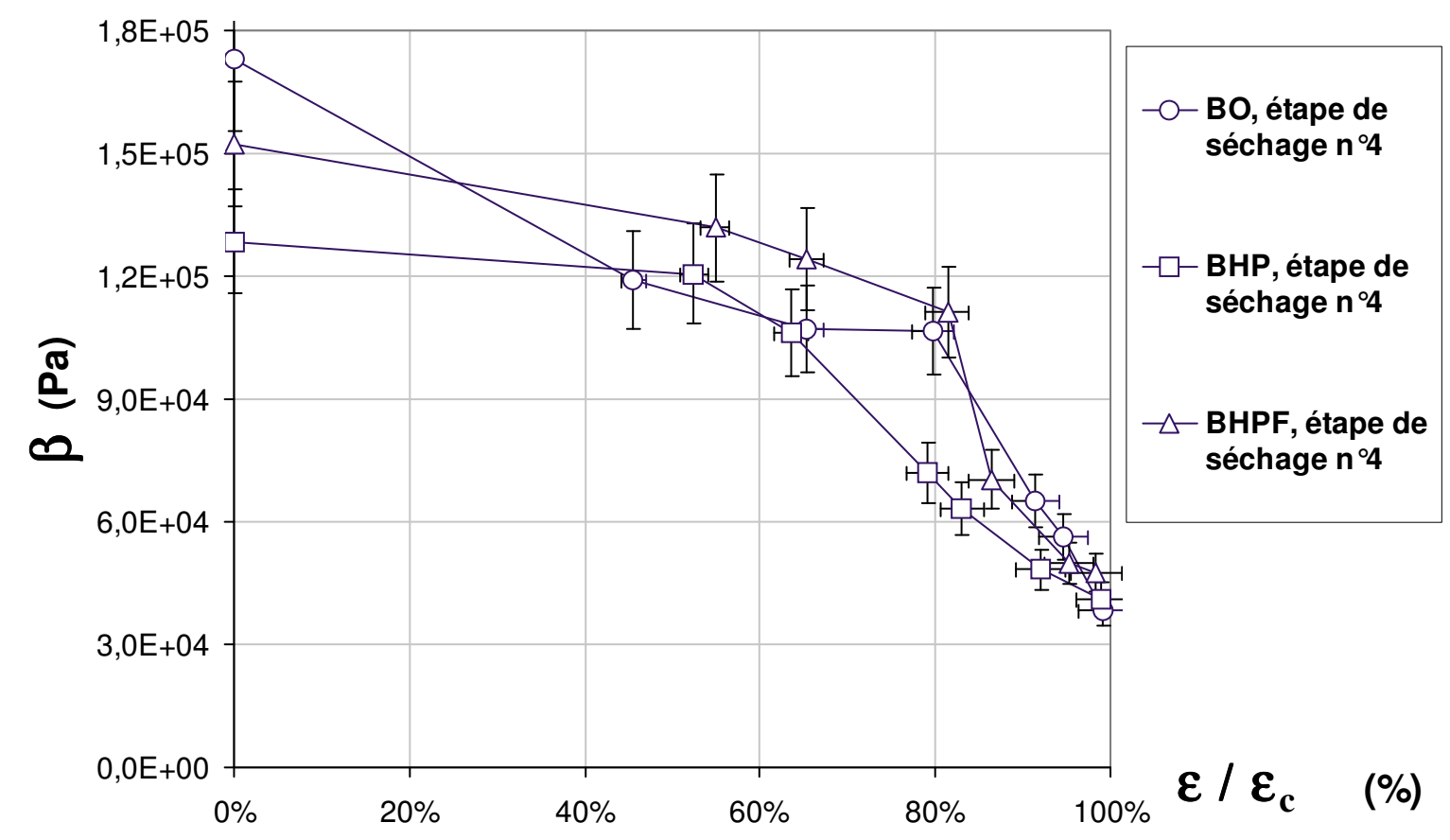

Figure 8. Evolution du coefficient $\beta$ selon le rapport de déformation $\varepsilon / \varepsilon_{c}$. Evolution of the coefficient $\beta$ versus the strain ratio $\varepsilon / \varepsilon_{\mathrm{c}}$.

\section{Effets du séchage}

L'augmentation de la perméabilité liée aux déformations subies a été analysée au cours du séchage des bétons étudiés.

\subsection{Accroissement absolu de perméabilité}

Les différences de perméabilité, $\mathrm{k}_{\mathrm{V}}(\mathrm{d})$ - $\mathrm{k}_{\mathrm{V}} \mathrm{O}$, représentées en Figure 9 augmentent avec le séchage. Les traits en pointillés sont tracés à partir des points dont la perméabilité a été approchée à partir de $\mathrm{k}_{\mathrm{A}}(\mathrm{cf}$. §4.1). La différence de perméabilité intrinsèque effective est en réalité légèrement supérieure.

Durant le séchage, les dépressions capillaires qui s'exercent dans le matériau font que ce sont d'abord les fissures et les pores les plus gros qui sont drainés en premier, tandis que de l'eau demeure toujours dans les capillaires les plus fins. Le diamètre moyen des capillaires saturés en eau qui ne participent pas à l'écoulement gazeux diminue avec le séchage. L'augmentation continue de la différence entre $\mathrm{k}_{\mathrm{V}}(\mathrm{d})$ et $\mathrm{k}_{\mathrm{V}} \mathrm{O}$ avec le séchage laisse supposer que l'endommagement produit est multiéchelle. 
De plus, pour un béton donné, l'augmentation de perméabilité entre les étapes successives de séchage est proportionnelle pour chaque seuil de déformation observé. La distribution de la taille des fissures engendrées est donc étalée et homogène quel que soit le seuil de déformation maximal atteint. D'ailleurs, pour les échantillons les plus endommagés, c'est à dire lorsque le rapport $\varepsilon / \varepsilon c$ tend vers 1 , les valeurs de $\beta$ pour les trois bétons étudiés sont très proches et évoluent peu avec le séchage (cf. §5.3). D'un béton à un autre, la densité de fissuration peut être différente, mais la section moyenne des fissures traversées par le gaz est du même ordre de grandeur.

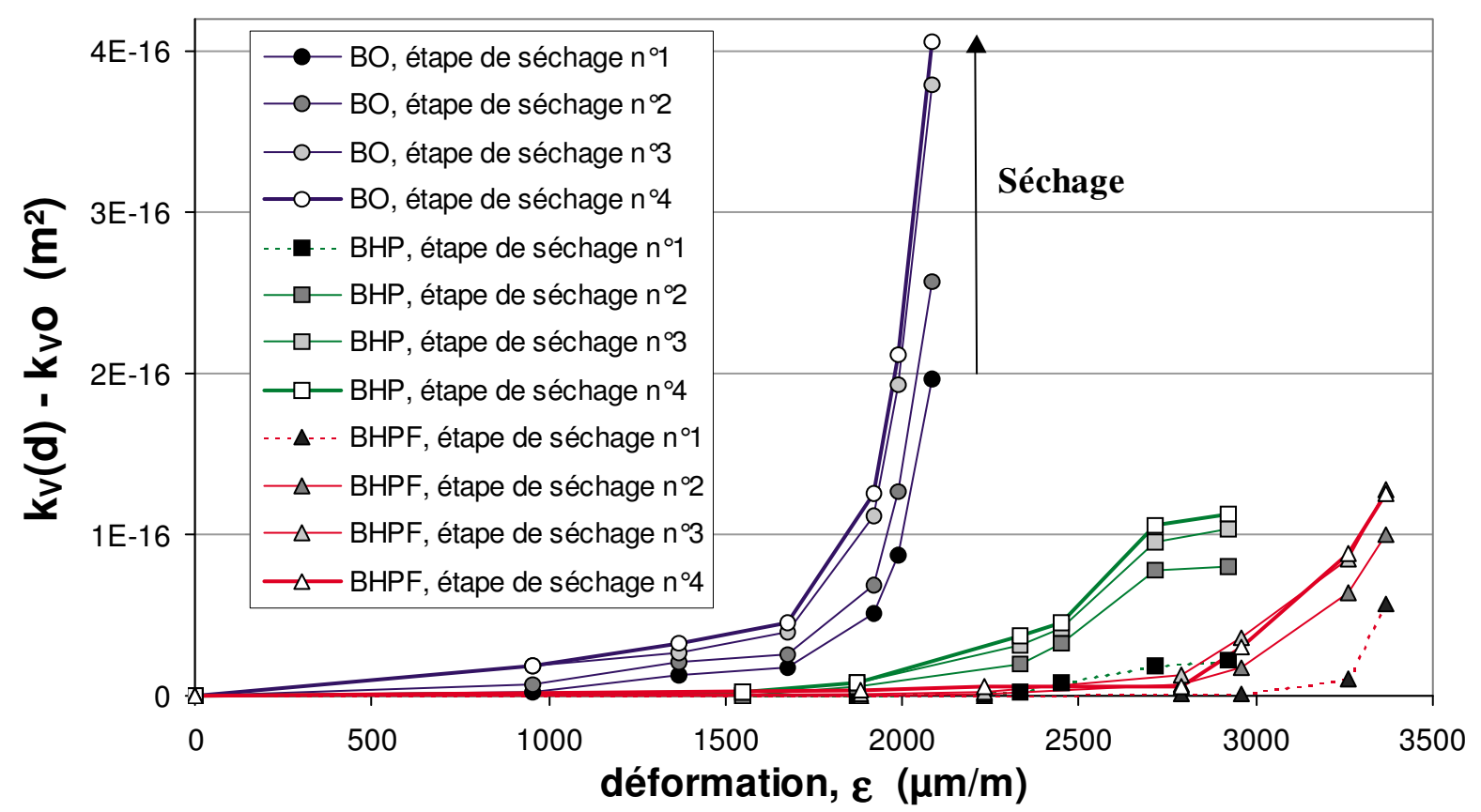

Figure 9. Accroissement absolu de perméabilité après chaque étape de séchage. Absolute increase in permeability after each drying stage

L'accroissement absolu de perméabilité tend à se stabiliser entre les deux derniers stades de séchage vers une valeur maximale, étant donné que les teneurs en eau d'une même série tendent vers la même valeur (cf. §3.1). Concernant les disques les plus endommagés, la différence entre $\mathrm{k}_{\mathrm{V}}(\mathrm{d})$ et $\mathrm{k}_{\mathrm{V}} \mathrm{O}$ est alors quatre fois plus grande pour le BO que pour les BHP. D'un point de vue quantitatif, pour des déformations proches de $\varepsilon_{c}$, la fissuration engendrée dans les BO est plus dommageable pour la perméabilité que celle engendrée dans les BHP. L'augmentation de la perméabilité est en effet quatre fois plus grande pour le BO que pour les BHP.

\subsection{Rapport d'accroissement de perméabilité}

Les flèches noires sur la Figure 10 donnent en exemple l'effet du séchage sur la perméabilité du BO et du BHPF. Le rapport d'accroissement de la perméabilité avec 
le séchage est plus sensible pour les bétons sains que pour les bétons endommagés. Cette tendance est particulièrement marquée pour les deux BHP.
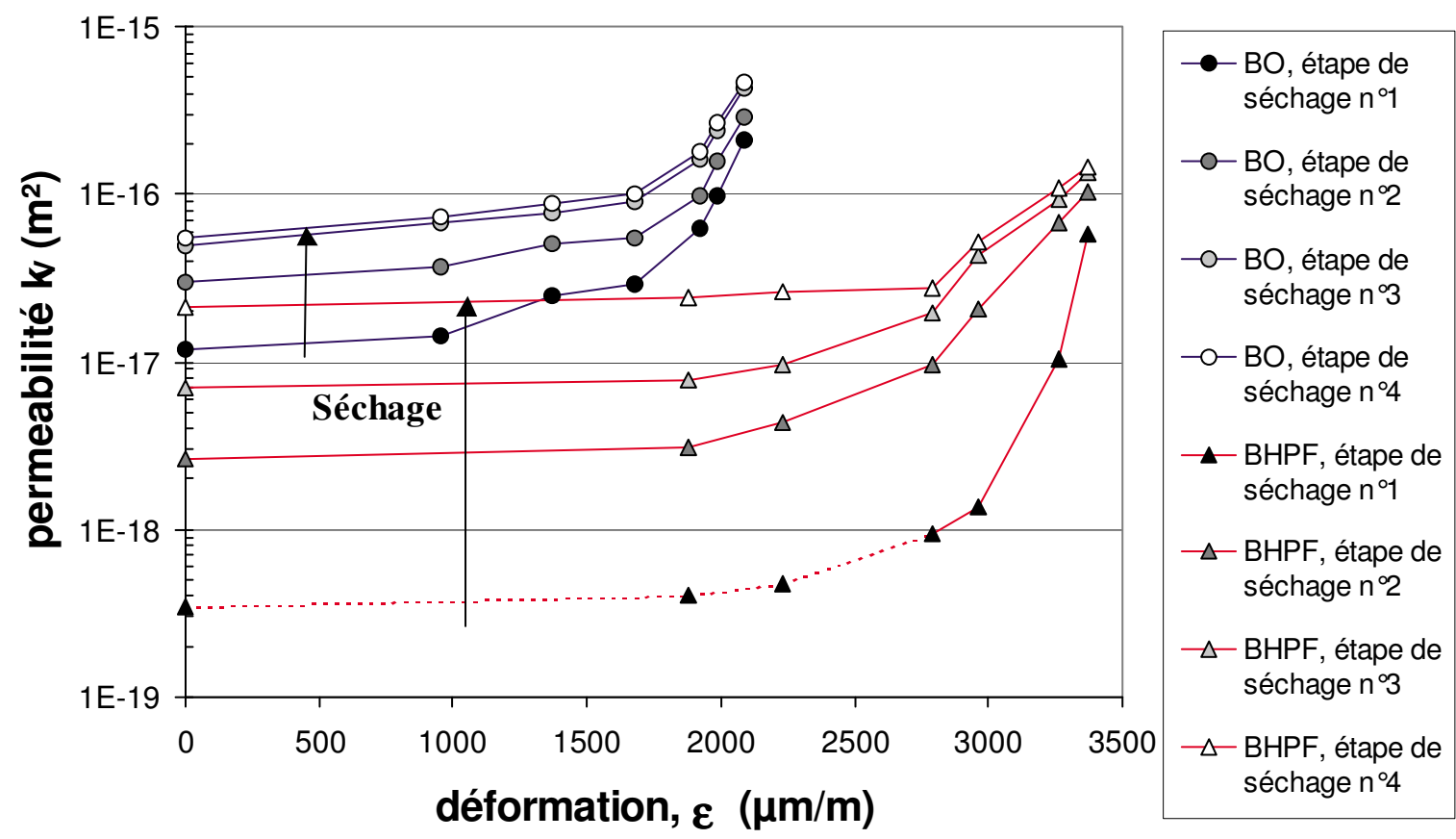

Figure 10. Perméabilité des $B O$ et des BHPF après chaque étape de séchage. Permeability of BO and BHPF after each drying stage

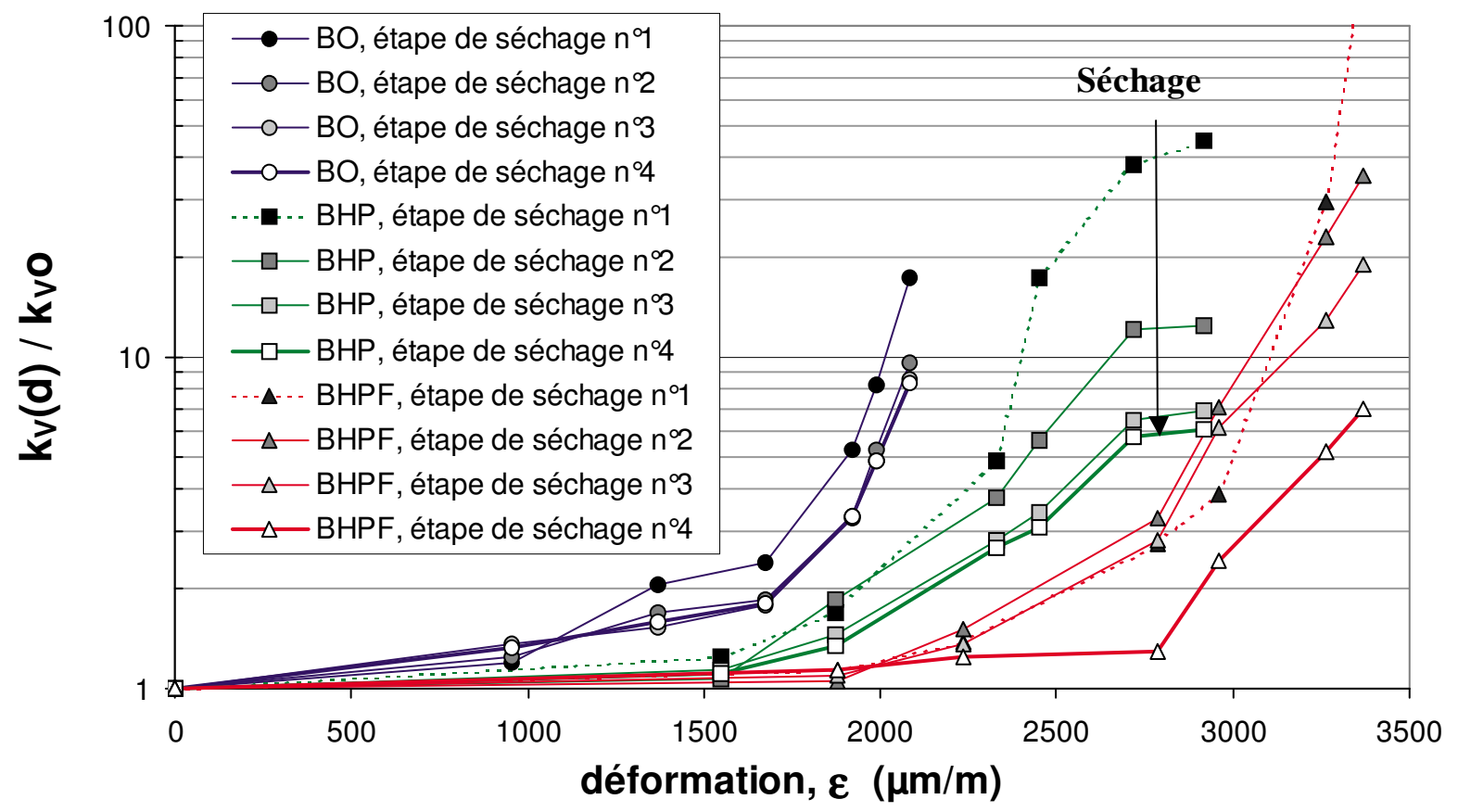

Figure 11. Rapport d'accroissement de la perméabilité après chaque étape de séchage. Increase ratio of permeability after each drying stage 
Le rapport d'accroissement de perméabilité, c'est à dire le rapport $\mathrm{k}_{\mathrm{V}}(\mathrm{d}) / \mathrm{k}_{\mathrm{V}} \mathrm{O}$ (voir Figure 11), tend donc à diminuer avec le séchage pour deux raisons :

- Premièrement, les disques endommagés présentent des microfissures qui augmentent leur diffusivité hydrique et de ce fait, leur cinétique de séchage (cf. §3.1). Après un séchage modéré, leur degré de saturation diminue selon leur endommagement et donc leur perméabilité au gaz augmente [JAC 98], [ABB 99]. Cependant, après l'étape de séchage $\mathrm{n}^{\circ} 2$, les différences de cinétique de séchage causées par l'endommagement n'interviennent quasiment plus sur les degrés de saturation, et donc, sur les mesures de perméabilité d'une série d'échantillons.

- Deuxièmement, durant la mesure de la perméabilité des disques endommagés, ce sont les réseaux de fissures qui contrôlent l'essentiel du flux gazeux à travers l'échantillon [GER 96], [TOR 99]. Comme les fissures sont drainées en premier, un séchage plus important ne permet que le séchage de fissures ou de pores plus fins qui ne participent qu'à une part mineure du flux global de gaz.

A la fin du séchage, comme précédemment, le rapport d'accroissement de la perméabilité tend à se stabiliser entre les deux derniers stades de séchage, vers une valeur minimum cette fois ci. Il présente par ailleurs, pour les trois bétons étudiés, une évolution similaire selon le rapport de déformation $\varepsilon / \varepsilon_{\mathrm{c}}$. De plus, en considérant la manifestation mécanique de l'endommagement, c'est à dire la perte de raideur du matériau ou la diminution relative du module d'élasticité, une similarité dans l'évolution des trois bétons a aussi été observée.

Il est à noter qu'après la première étape de séchage, le rapport $\mathrm{k}_{\mathrm{V}}(\mathrm{d}) / \mathrm{k}_{\mathrm{V}} \mathrm{O}$ effectif est encore supérieur à celui tracé en pointillé pour les BHP à partir des valeurs de perméabilité apparente (cf. § 4.1). Dans ce cas, ces rapports d'accroissement important peuvent alors être attribués aux deux raisons évoquées précédemment, dans des mesures différentes. En supposant que tous les échantillons soient à $90 \%$ de saturation, la partie saine des échantillons serait quasi-imperméable et tout le flux gazeux serait alors canalisé par le réseau éventuel de fissures, même si ce dernier contenait encore de l'eau adsorbée (cas du flux de gaz $n^{\circ} 2$, Figure 4). Ce flux gazeux, aussi faible soit-il, provoquerait alors un rapport d'accroissement très important de la perméabilité globale de l'échantillon. C'est pourquoi de manière générale les résultats expérimentaux montrent que le degré de saturation des échantillons est un facteur qui, suite aux endommagements éventuels du matériau, amplifie à lui seul le rapport d'accroissement de la perméabilité.

\subsection{Flux de gaz pour une pression différentielle donnée}

Après chaque étape de séchage, les résultats montrent qu'il n'y a pas d'évolution significative du coefficient $\beta$, c'est à dire du rapport des débits $\mathrm{Q}_{\mathrm{glis}} / \mathrm{Q}_{\mathrm{visq}}$ (cf. §2.4, équation [3]), particulièrement pour les éprouvettes endommagées. Deux phénomènes ayant des effets opposés sur la variation de $\beta$ se produisent au cours du séchage : 
- Le drainage des pores et microfissures les plus fins augmente la part des écoulements par glissement.

- La disparition des couches d'eau adsorbées sur les parois des fissures, diminue les écoulements par glissement qui y prenaient place tout en augmentant la proportion globale du débit des écoulements visqueux.

Les comparaisons relatives de perméabilité, apparente ou intrinsèque, évoluent donc dans des proportions similaires avec le séchage du béton.

\section{Conclusion}

Un endommagement par compression en phase pré-pic augmente sensiblement la perméabilité d'un béton. Cette augmentation de perméabilité est directement liée aux déformations maximales subies durant le chargement, indépendamment du nombre de cycles de charge. Pour des déformations proches de la déformation maximale au pic, la perméabilité intrinsèque est augmentée d'un ordre de grandeur.

Ces augmentations de perméabilité après déchargement sont relatives à la formation de microfissures qui ne se referment pas complètement, d'ailleurs la variation du coefficient $\beta$ atteste d'un élargissement du rayon moyen des pores participant à l'écoulement global. Par ailleurs, il est clair que, sous contrainte, les différences de perméabilité étudiées seraient davantage amplifiées.

En fonction du paramètre de déformation $\varepsilon / \varepsilon_{\mathfrak{c}}$, l'évolution de la perméabilité des trois bétons étudiés est similaire (augmentation très nette au delà du seuil $\varepsilon / \varepsilon_{\mathrm{c}}=0.8$ ). Cependant la perméabilité des BO est toujours supérieure à celle des BHP qui, pourtant, supportent des déformations nettement supérieures.

La perméabilité au gaz peut donc efficacement distinguer un béton endommagé d'un béton sain. Les comparaisons de perméabilité dépendent du degré de saturation en eau du béton et peuvent être relatives ou quantitatives, selon les paramètres d'évolution considérés vis à vis de la durabilité.

- D'un point de vue quantitatif, la diminution du degré de saturation en eau augmente l'accroissement absolu de la perméabilité.

- D'un point de vue comparatif, la diminution du degré de saturation en eau réduit l'accroissement relatif de la perméabilité.

Pour une étude comparative de perméabilité au gaz, le contrôle de l'état hydrique du béton testé est donc essentiel.

\section{Bibliographie}

[ABB 99] AbBas A., CARCASSES M., Ollivier J-P., " Gas permeability of concrete in relation to its degree of saturation ", Materials and Structures, vol 32, 1999, pp. 3-8. 
[ABE 97] ABEBE DinKu, ReINHARDT H.W., " Gas permeability coefficient of cover concrete as a performance control ", Materials and Structures, vol 30, 1997, pp. 387-393.

[BOU 99] Boulay C., Le Maou F., Renwez S., "Quelques pièges à éviter lors de la détermination de la résistance et du module à la compression sur cylindres de bétons ”, Bulletin des Laboratoires des ponts et chaussées, n²20, 1999, pp. 63-74.

[GER 96] Gérard B., Breysse D., Ammouche A., Houdusse O., Dirdry O., “Cracking and permeability of concrete under tension ", Materials and Structures, vol 29, 1996, pp. 141151.

[HEA 97] HEARN N., G. LOK, “ Measurement of permeability under uniaxial compression - A test method ", ACI Materials Journal, vol 95, n6, 1997, pp. 691-694.

[HEA 99] HEARN N., " Effect of shrinkage and load-induced cracking on water permeability of concrete ”, ACI Materials Journal, vol 96, n², 1999, pp. 234-241.

[JAC 98] JACOBS F., “Permeability to gas of partially saturated concrete", Magazine of Concrete Research, vol 50, n², 1998, pp. 115-121.

[KER 91] KERMANi A., "Permeability of stressed concrete", Building Research and Information, vol 19, $\mathrm{n}^{\circ} 6,1991, \mathrm{pp} .360-366$.

[KOL 89] KolLeK J.J., “ The determination of the permeability of concrete to oxygen by the Cembureau method - a recommendation ", Materials and Structures, vol 22, 1989, pp. 225-230.

[PIC 00] PiCAndet V., Khelidj A., Bastian G., “Effect of mechanical damaging on gas permeability of concrete", $2^{\text {nd }}$ International Symposium on Cement and Concrete Technology, Istanbul, Turkey, 6-10 September 2000, pp. 322-331.

[QUE 97] Quenard D., CARCASSES M., “Les résultats des essais croisés AFREM : Perméabilité", Compte-rendu des journées techniques AFPC-AFREM «Durabilité des bétons », Toulouse, 11-12 décembre 1997, pp. 41-58.

[SAM 92] H.R. SAMAHA, K.C. HOVER "Influence of microcracking on the mass transport properties of concrete ”, ACI Materials Journal, vol 89, n4, 1992, pp. 416-424.

[SUG 96] SugiYama T., Bremner T.W., Holm T.A., “ Effect of stress on gas permeability in concrete ”, ACI Materials Journal, vol 93, n5, 1996, pp. 443-450.

[TOR 99] Torrenti J-M., Didry O, Ollivier J-P.,Plas F., La dégradation des bétons, couplage fissuration-dégradation chimique, Editions Hermès, Paris, 1999.

[WAN 97] WANG K., JANSEN D.C., SHAH S.P. "Permeability study of cracked concrete”, Cement \& Concrete Research, vol 27, n³, 1997, pp. 381-393.

[YSS 97] YSSORCHE M-P, “Importance de l'état hydrique du matériau sur la mesure de la perméabilité au gaz", Compte-rendu des journées techniques AFPC-AFREM «Durabilité des bétons », Toulouse, 11-12 décembre 1997, pp. 25-39. 